\title{
Game Changing: NASA's Space Launch System and Science Mission Design
}

\author{
Stephen D. Creech \\ National Aeronautics and Space Administration \\ Space Launch System Program \\ Marshall Space Flight Center, AL 35812 \\ 256-544-9365 \\ Stephen.D.Creech@nasa.gov
}

\begin{abstract}
NASA's Marshall Space Flight Center (MSFC) is directing efforts to build the Space Launch System (SLS), a heavy-lift rocket that will carry the Orion Multi-Purpose Crew Vehicle (MPCV) and other important payloads far beyond Earth orbit (BEO). Its evolvable architecture will allow NASA to begin with Moon fly-bys and then go on to transport humans or robots to distant places such as asteroids and Mars.

Designed to simplify spacecraft complexity, the SLS rocket will provide improved mass margins and radiation mitigation, and reduced mission durations. These capabilities offer attractive advantages for ambitious missions such as a Mars sample return, by reducing infrastructure requirements, cost, and schedule. For example, if an evolved expendable launch vehicle (EELV) were used for a proposed mission to investigate the Saturn system, a complicated trajectory would be required - with several gravity-assist planetary fly-bys - to achieve the necessary outbound velocity. The SLS rocket, using significantly higher characteristic energy (C3) energies, can more quickly and effectively take the mission directly to its destination, reducing trip time and cost.
\end{abstract}

As this paper will report, the SLS rocket will launch payloads of unprecedented mass and volume, such as "monolithic" telescopes and in-space infrastructure. Thanks to its ability to co-manifest large payloads, it also can accomplish complex missions in fewer launches. Future analyses will include reviews of alternate mission concepts and detailed evaluations of SLS figures of merit, helping the new rocket revolutionize science mission planning and design for years to come.

\section{TABLE OF CONTENTS}

1. INTRODUCTION ......................................................... 1

2. Program OVERVIEW .......................................... 2

3. Program Status - Steady Progress ............ 6

4. PlatFORM FOR EXPANDED MISSIONS............... 9

5. SUMMARY ..................................................... 12

REFERENCES ...................................................... 13

BIOGRAPHY ........................................................ 13

\section{INTRODUCTION}

NASA's SLS is a key cornerstone of a flexible path approach to space exploration. This approach, part of the NASA Authorization Act of 2010, opens up vast opportunities for new human exploration destinations, including cis-lunar, near-Earth asteroids, and Mars. [1]

A flexible path, in the words of the 2009 Augustine Commission in its review of U.S. human spaceflight plans, represents an exploration strategy that will allow humans to learn how to live and work in space, to visit small bodies, and to work with robotic probes on planetary surfaces, and will provide the public and other stakeholders with a series of interesting "firsts" to keep them engaged and supportive. [2]

The SLS architecture, scheduled for Preliminary Design Review in 2013 and for a first launch in 2017, reflects this flexible path strategy. Since 2010, the Agency has been moving forward with a space exploration program designed to carry human beings beyond low-Earth orbit (LEO) for the first time since 1972, when the Apollo Program concluded its sixth and final landing on the Moon.

The NASA Authorization Act of 2010 included development of the heavy-lift SLS, the Orion MPCV, and Ground Systems Development and Operations (GSDO) as core elements of NASA's human space exploration architecture. The Act specified initial and evolved SLS capabilities to support early and future missions beyond LEO. The SLS vehicle would initially lift 70 metric tons (t) to LEO and be evolvable to $130 \mathrm{t}$ or more; and lift the Orion MPCV. 


\section{Program Overview}

NASA and the U.S. Congress formally announced the development of SLS on September 14, 2011. This specific architecture was selected largely because it utilizes an evolvable development approach, which allows NASA to better reconcile typical development cost curves with the reality of a non-increasing budget.

In order to do this, the architecture leverages existing capabilities and lowers development costs by using such assets as Saturn- and Shuttle-derived engines, and, for the initial configuration missions, Shuttlederived solid motors. And, for the first time, legacy systems include propulsion and control technologies developed in this century. The new launch capability would leverage experience, including contractual experience, regardless of heritage. Existing contract vehicles were included to benefit from past investments, and acquisition plans reflect this direction.

While the new launch capability will require new development, such as new spacecraft and payload adapter and fairing designs, it would be composed, to the greatest extent practicable, of proven systems that possess performance history.

The design selected by the Agency was closest, from among hundreds considered, to fitting within a nonincreasing annual budget profile, while meeting system-level requirements (Figure 1). This was due to several factors, such as the rocket's relatively simple design (i.e., minimum number of hardware elements to achieve the desired performance) and the plan to start with existing Agency hardware assets and other elements that were well into the development cycle.

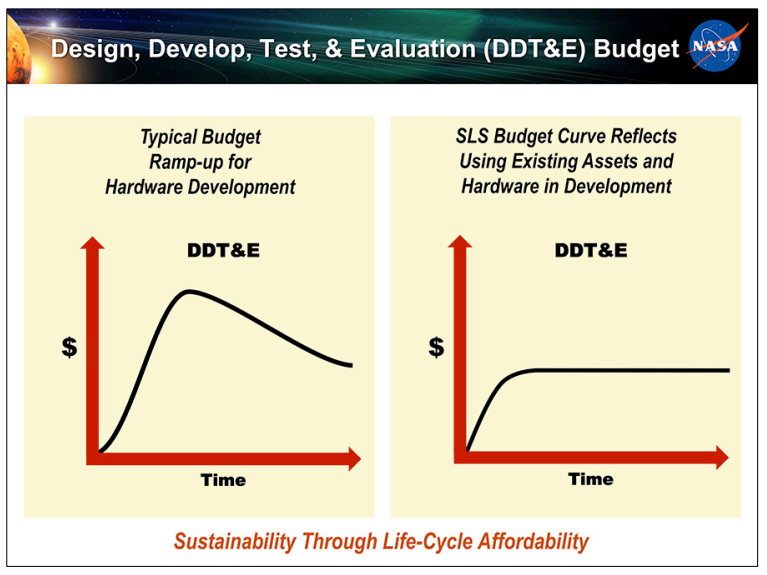

Figure 1 - Development budget curve
This strategy responds to a nontraditional design, development, test, and evaluation (DDT\&E) budget curve, which keeps the system affordable and sustainable over the decades that an endeavor such as this will span. As such, production and operations costs are also considered as independent variables in trade studies, along with nonrecurring development costs.

In accordance with the 2010 legislation-and to leverage NASA's investments in heritage systems such as the Space Shuttle and the Constellation Program-NASA is using several existing or indevelopment systems to reduce the SLS's DDT\&E cost, risk, and schedule.

The benefits of this approach are multiple. Perhaps the greatest budgetary strain on a new launch vehicle project is the multiple, parallel development efforts it requires. Leveraging heritage systems reduces the number of parallel developments needed for SLS; the core stage is the only major initial-configuration element that follows a traditional development budget curve.

In addition, using legacy hardware and systems also reduces the costs for those elements. For example, NASA already has in inventory enough RS-25 core stage engines for the first four SLS flights; representing a significant savings for the program.

The SLS will have the largest payload lifting capacity of any launch vehicle previously manufactured in the U.S., allowing for accommodation of many mission profiles, starting with Orion MPCV missions for lunar fly-by and high lunar orbit and eventually deep space near-Earth asteroid (NEA) and Mars missions that extend human presence across the solar system (requiring $\sim 130 \mathrm{t}$ ). The SLS will also accommodate Orion MPCV missions to LEO for system test and checkout and as a backup access to the International Space Station (ISS), including transport of replacement ISS modules.

Other mission profiles that can be supported by the SLS include science-based missions for deep space astronomy and solar system exploration. The SLS payload capability will enable a new generation of planetary, Earth, and heliophysics science missions. SLS will also be capable of supporting commercialbased missions and missions supporting other Government agencies, including sending larger objects into LEO, such as commercial space stations. 
The SLS's unprecedented mass capability is also complemented by the largest payload volume ever provided by a single launch vehicle (Figure 2). Set for first flight in 2017, the $70 \mathrm{t}$ configuration will lift more than 154,000 pounds and will provide 10 percent more thrust than the Saturn V rocket. This mass and volume capability to LEO enables a host of new scientific and observation platforms, such as telescopes, satellites, and planetary and solar missions, as well as provides the lift for future large in-space infrastructure missions, such as space-based power and mining, Earth asteroid defense, and propellant depots.

The SLS will replace and exceed the transportation capability of the historic Saturn V, both in terms of volume and mass. As such, it will be an unmatched national asset for exploration, science, national security, and commercial payloads.

With the retirement of the Space Shuttle Program and cancellation of the Constellation Program, the Agency is funding commercial activities that will yield a commercial cargo capability during the transition to NASA's new human space exploration architecture.
As shown in Figure 3, SLS complements NASA's commercial crew and cargo initiatives, which are creating a supply line to and from the International Space Station and LEO. In contrast, the evolvable SLS super heavy-lift rocket is designed for BEO missions, and will be a platform for continuing America's tradition of human space flight, as well as providing the capability to launch entirely new science missions and other missions of national and international importance.

By maintaining a reasonable number of launches per mission, simplifying on-orbit operations, maximizing mission reliability, and offering a very large payload volume, SLS could enable or significantly enhance a large class of space missions that would allow prospective mission planners the ability to build robust payloads with margins significantly higher than the industry norm. The SLS investment can be leveraged for many types of missions, including deep space exploration, planetary landers, human habitats, great observatories, space solar power, outer planet missions, and Department of Defense (DoD)/National Reconnaissance Office (NRO) payloads.

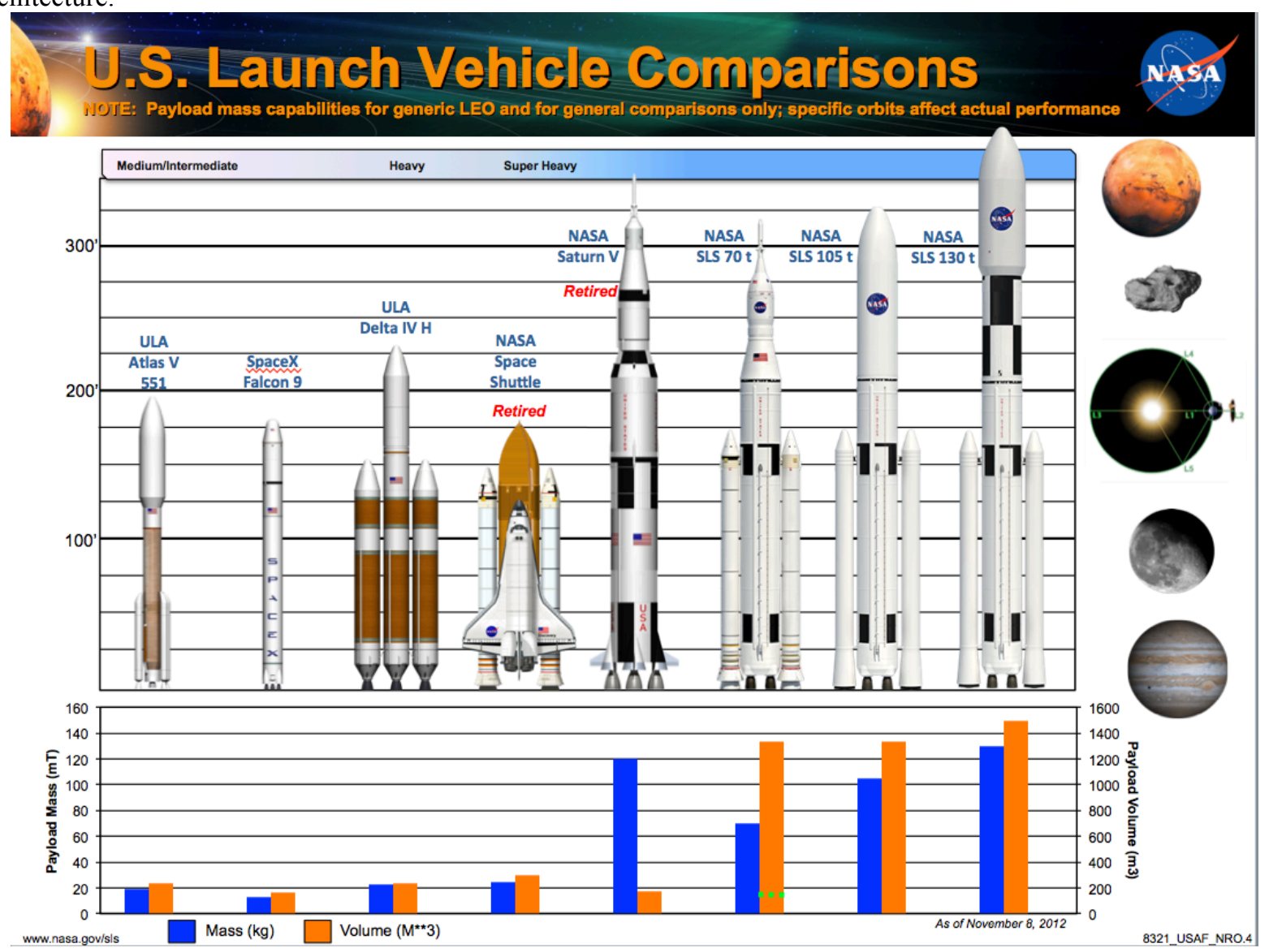

Figure 2 - Launch vehicle comparisons 


\section{The Future of Exploration}

"This expanded role for the private sector will free up more of NASA's resources to do what NASA does best - tackle the most demanding technological challenges in space, including those of human space flight beyond low-Earth orbit."

Mars

— John P. Holdren, Science and Technology Assistant to the President The White House, May 22, 2012
$34,600,000 \mathrm{mil}$ $55,700,000 \mathrm{~km}$

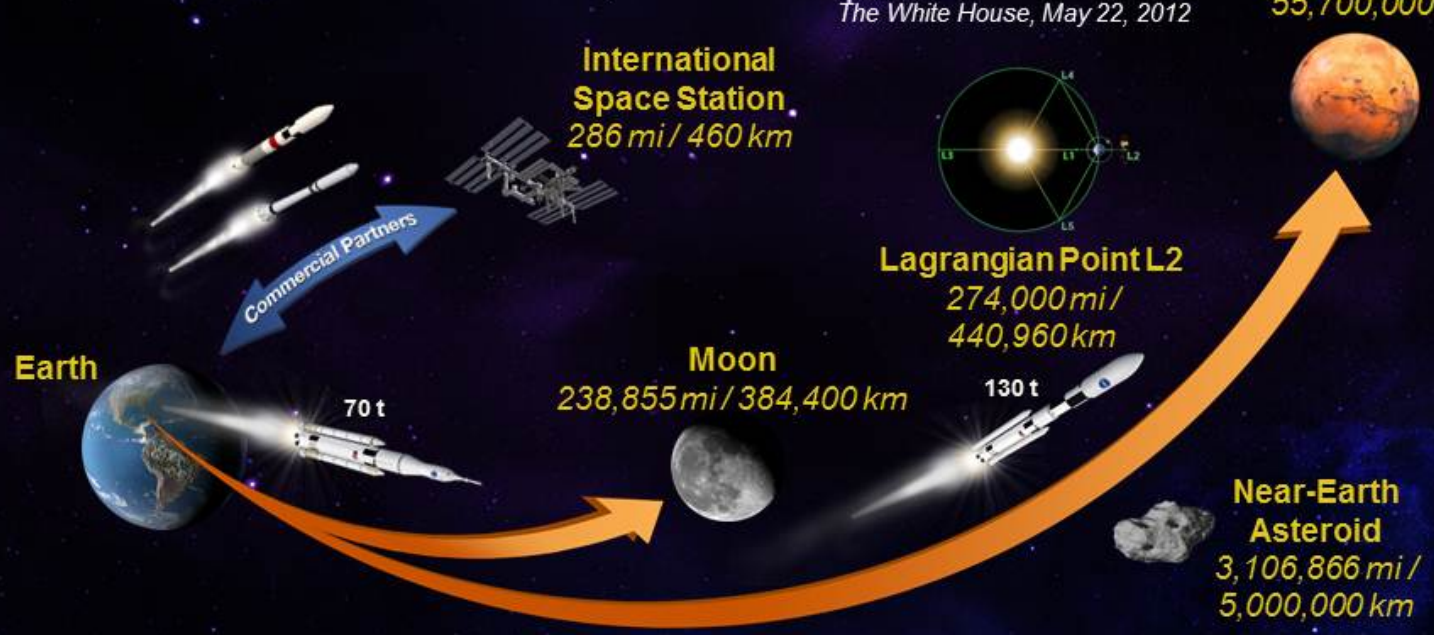

"My desire is to work more closely with the human spaceflight program so we can take advantage of synergy. We think of the SLS as the human spaceflight program, but it could be hugely enabling for science.

- John Grunsfeld, Associate Administrator NASA Science Mission Directorate Nature, Jan 19, 2012

Figure 3 - The evolved SLS rocket is designed for missions beyond Earth's orbit

\section{SLS Decision Rationale}

Ultimately, a carefully considered architecture decision met policy and law while leveraging development progress, the U.S. aerospace industry's workforce experience, existing assets, and unique infrastructure. The flexible path/capability-driven framework decision for the SLS vehicles maintains U.S. leadership in liquid oxygen/liquid hydrogen (LOX/LH2) propulsion technology by leveraging experience within the Agency and its industry partners on the RS-25 core stage engines and the J$2 \mathrm{X}$ upper stage engine. The capability-driven framework establishes a fixed central design path, with the logical use of existing strengths in design and modern manufacturing approaches. It also harnesses the existing knowledge base, skills, infrastructure, workforce, and industrial base for state-of-the-art systems.

Minimizing unique configurations during vehicle development, the evolutionary path to a $130 \mathrm{t}$ vehicle allows for incremental development, enabling progress to be made even within constrained budgets, while also allowing for early flight certification for the Orion MPCV. Thus, SLS may be configured for Orion or other large cargo payloads, providing a flexible/modular design and a system for varying launch needs.

\section{Safety, Affordability, Sustainability}

SLS is being designed with three key principles in mind: safety, affordability, and sustainability.

Safety is a top priority for human-rated systems. Programmatic and technical functions of the program will be implemented in a manner that meets acceptable safety standards and requirements for all phases of the program life cycle. Program safety must be maintained while working toward an affordable and sustainable solution. Basing the SLS design on highly reliable human-rated propulsion elements established a foundation of safety upon which the 
program is being developed in close collaboration with safety and mission assurance representatives.

In the current policy and economic environment, affordability means designing and conducting work within a flat budget for the foreseeable future. This will be achieved by maximizing the use of common elements and existing assets, infrastructure, and workforce while also providing competitive opportunities to develop new systems. Sustainability is the ability of the SLS Program to remain affordable across multiple years and to support multiple missions. Again, SLS will ensure sustainability by leveraging common manufacturing facilities, tooling, materials, and processes/practices; experienced employees; supply chain and industry base; transportation logistics; ground systems/launch infrastructure; and propellants, all while living within its appropriated budget.

In a resource-constrained environment, SLS must fit within its resource envelope (e.g., budget, workforce, and facilities). The SLS Program is incorporating affordability initiatives and lean practices as it strives to deliver a safe, affordable, flexible, and sustainable long-term national capability.

Built on the fundamental principle that affordability begins with accountability, the evolvable development approach has adopted an innovative testing philosophy to meet requirements. For example, additional qualification testing will only be undertaken where there is a significant shortfall in the original item qualification limits and where qualification by other means is not possible. Significant cost savings can be realized by focusing development efforts on credible failure modes using probabilistic methods rather than utilizing development approaches that baseline multiple safety margins across multiple disciplines Further, a 27.5feet (8.4-meter) common diameter for both core and upper stages could yield a 20 -percent reduction in DDT\&E costs, a simplified design, common tooling and facilities, and a reduced number of suppliers and associated oversight.

SLS booster design and development activities have also employed a value stream mapping process to evaluate production of 5-segment solid rocket boosters, resulting in hundreds of changes that eliminate sources of waste and hardware moves and improve cycle time by nearly half. [3]

And while effectively managing requirements and leveraging existing capabilities, including LOX/LH2 propulsion infrastructure, manufacturing facilities, and launch sites, the evolvable development approach is infusing new design solutions for affordability. For instance, a risk-based Government insight/oversight model comes with the realization that significant cost can be driven by the level of Government-contractor interaction, the number of products and deliverables requested through the procurement process, and the number and type of requirements. This new insight/oversight model is a significant change to the philosophy and culture of previous NASA human spaceflight programs.

Right-sized documentation and standards will result in a reduction in the majority of data requirements and program documents from past projects, as well as better implementation of industry practices and tailored NASA standards. Lean, integrated teams with accelerated decision making provide simple, clear technical interfaces with contractors; an integrated systems engineering and integration (SE\&I) organization; empowered decision makers at all levels; fewer control boards and streamlined change processes; use of heritage hardware and manufacturing solutions; and the ability to maintain adequate management budget reserves controlled at lower levels.

\section{Architecture Description}

The SLS Program is comprised of multiple elements that will be developed and integrated into an evolved launch vehicle designed to enable exploration missions beyond LEO. As the orbital delivery system for this human and cargo space exploration architecture, the SLS Program will provide the necessary functions of crew and cargo launch; vehicle staging; vehicle communication with the Orion MPCV, GSDO, and U.S. Air Force Eastern (launch) Range; vehicle fault management; LEO or trajectory insertion; and payload separation at the destination orbit or trajectory.

The reprioritization of affordability as a key measure of program success has led NASA to reevaluate past heavy-lift launch vehicle studies to focus on design concepts that can support a variety of mission sets defined by evolving space exploration architecture capabilities within a capability-driven framework. Accordingly, NASA's system architecture for exploring space is developed incrementally to support missions with increasing needs for system capability. The SLS architecture is being developed around a low rate of missions, so any additional missions could yield further affordability benefits. 
As shown in Figure 4, the $70 \mathrm{t}$ configuration will consist of the 27.5-foot (8.4-meter) diameter core stage powered by four RS-25 LOX/LH2 engines, which formerly powered the Space Shuttle and builds on the U.S. state of the art in liquid propulsion. The core stage will be flanked by two 5 -segment solid rocket boosters (SRBs) - a more powerful version of the 4-segment boosters used on Shuttle and developed originally for the Ares launch vehicles. For the first two SLS missions, a commercial Interim Cryogenic Propulsion Stage (ICPS) will be used to propel the Orion spacecraft from LEO toward the Moon. The ICPS will provide the in-space propulsion needed until NASA's missions require the J-2X upper stage engine for larger payloads launched BEO.

The SLS block upgrade strategy, shown in Figure 4, reflects the scope of the capability-driven framework by incrementally and practically increasing the performance of the launch vehicle one block at a time. The performance thresholds for the SLS vehicles are achieved respectively through the development of blocks. This strategy relies on the use of existing technology, followed by new boosters, and eventually an upper stage for Block 2. The upgrade process will also depend on improving processes and the modification of other components, such as engines and materials. The SLS Program also recognizes that as new technologies are realized, the configuration of future blocks may be revised.

\section{Program Status - Steady Progress}

Just 1 year after program formulation, within a constrained budgetary environment and with a reduced workforce, SLS has made significant programmatic and hardware progress toward its first flight in 2017. It has successfully completed its System Requirements Review/System Definition Review (SRR/SDR) and received permission to move forward toward its Preliminary Design Review (PDR), currently scheduled for 2013.

While the SLS team is making swift progress on the initial configuration and building a solid baseline, it is also looking ahead to enhance and upgrade future configurations of the heavy lift vehicle. The NASA Research Announcement (NRA) for the Advanced Booster Engineering Demonstration and Risk Reduction Efforts is spurring competition that will empower entirely new exploration for missions of national importance. NASA has awarded three contracts-Alliant Tech Systems (ATK), Dynetics, and Northrop Grumman-to develop engineering demonstrations and/or risk reduction advanced booster concepts for the evolved rocket. [4]

NASA has also selected 26 proposals from academia and industry for advanced development activities for SLS. These proposals, in response to an SLS Advanced Development NRA, seek innovative and

\section{SLS Block Approach}

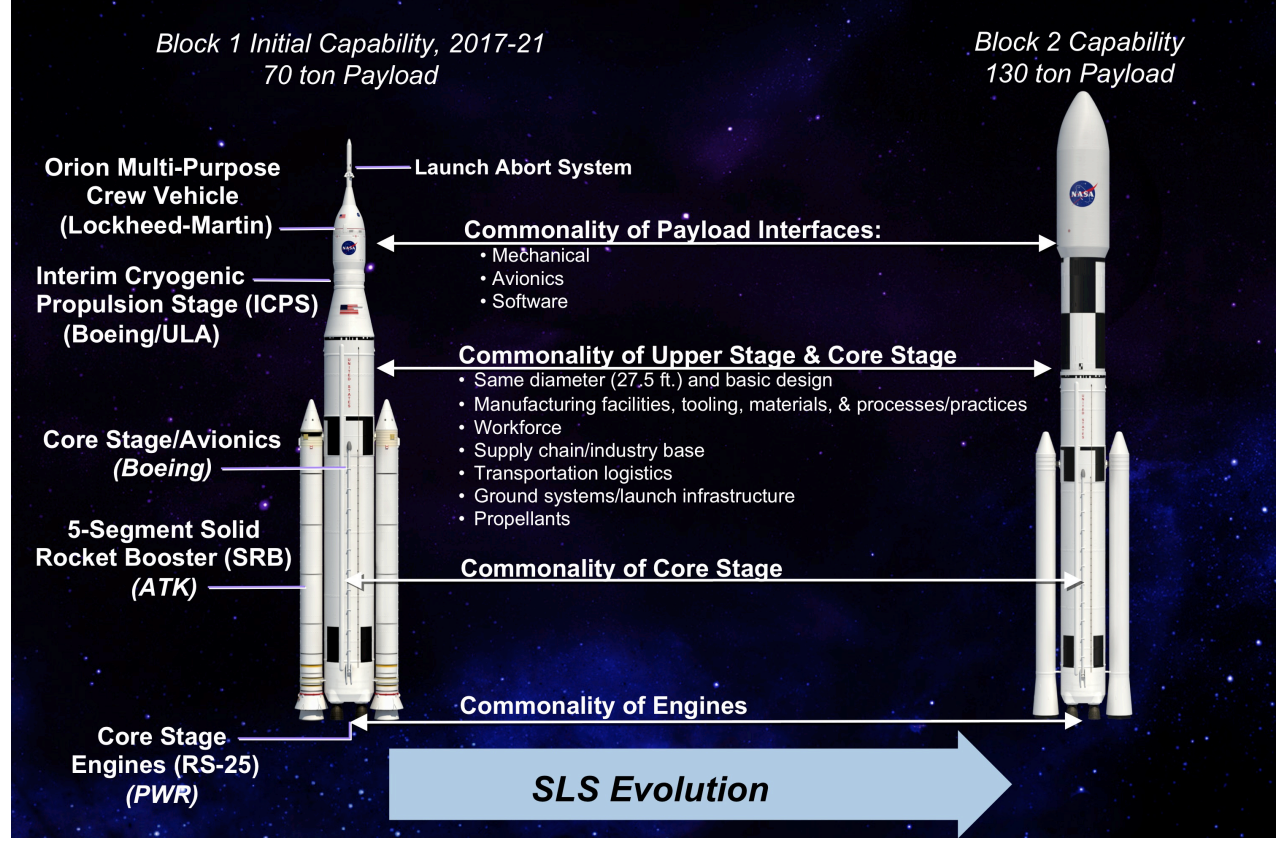

Figure 4 - SLS block evolution 
affordable solutions to evolve the launch vehicle from its initial configuration to its full lift capacity. The proposals cover a wide variety of areas, including concept development, trades and analyses, propulsion, structures, materials, manufacturing, and avionics and software. These proposals will help NASA optimize affordability while integrating mature technical upgrades into future vehicles. Formal contract awards will follow further negotiations between NASA and selected organizations. [5]

The mix of contract mechanisms SLS is using will help NASA take advantage of the experience of legacy systems while also allowing the Agency to "spin in" new ideas and technologies to help improve performance and reduce cost. It also addresses longlead items and infuses capital into the aerospace industry.

SLS has also made significant software and hardware progress in its first year of development, as summarized below.

\section{Avionics and Software}

The SLS avionics team is fine-tuning flight software, using test-bed computers received ahead of schedule from The Boeing Company in the MSFC Systems Integration Laboratory. Availability of the platform early in the engineering development phase allows NASA programmers more development time. Existing systems from communications and Global Positioning System (GPS) satellites are also being upgraded to provide the highest processing capability available.

\section{Hardware}

Use of legacy technology has provided a significant boost to the development of the vehicle's propulsion systems. SLS began development with its RS-25 core stage engines already at a Technology Readiness Level (TRL) of 9 (flight proven), and its other propulsion systems are currently at TRL 6 (qualification testing). [6]

ATK has completed three full-scale static tests of 5segment development motors for the booster systems (Figure 5). The SLS Boosters element, in conjunction with ATK, has also completed casting for the five segments of the qualification motor-1 (QM-1), scheduled for testing in spring 2013. The QM-1 test will round out the test series necessary to qualify the 5-segment booster for flight.

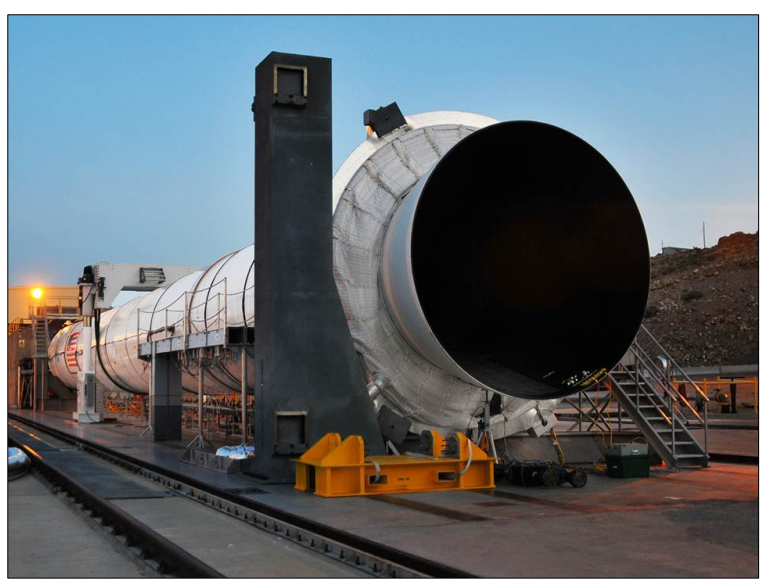

Figure 5 - A 5-segment booster development motor on the test stand

The first SLS-produced flight hardware, the MPCV Stage Adapter (MSA), has been manufactured (Figure 6) and will fly as part of the first exploration flight test (EFT-1) of an Orion test article in 2014 on a Delta IV rocket. This same MSA design will be used on the SLS Block 1.

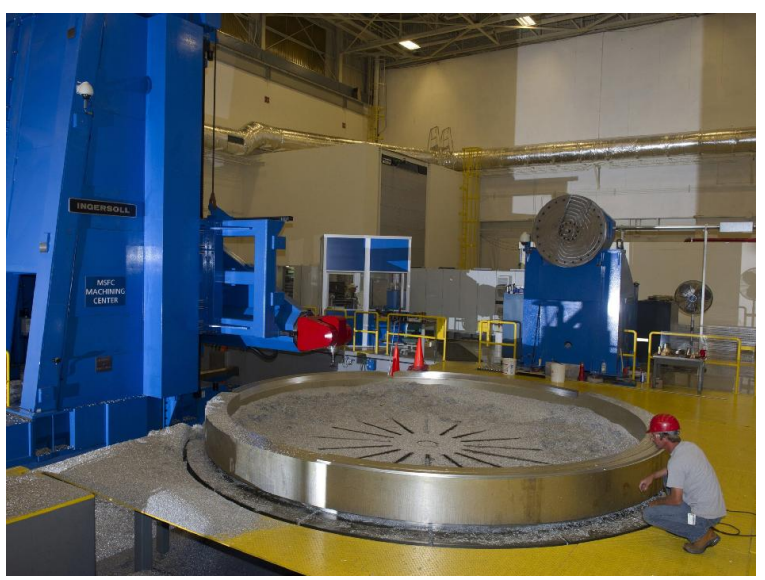

Figure 6 - MSA pathfinder hardware for EFT-1 at MSFC

Four complete sets (16 engines) of Pratt \& Whitney Rocketdyne (PWR) RS-25 core stage engines were delivered to Stennis Space Center (SSC) in April 2012, along with much of their related propulsion subsystems (Figure 7).

The J-2X upper stage engine, a modernized, more powerful version of the Saturn V's J-2 engine, is nearly finished with its development process. Engineers have completed 21 tests and over 2,700 seconds of hot-fire time at SSC. The engine attained 100 percent power in just its fourth test and later became the fastest U.S. rocket engine to achieve a full-flight duration test, hitting that 500-second mark in its eighth test (Figure 8). 


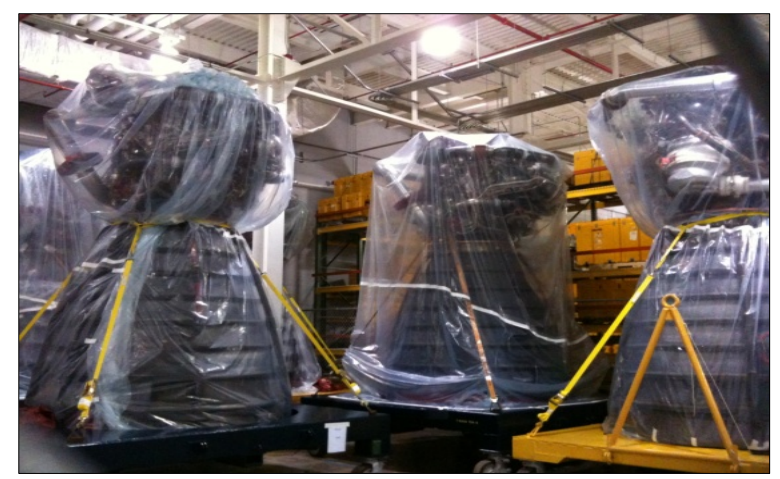

Figure 7 - RS-25 core stage engines in storage

NASA recorded another first during a 40 -second test of the engine in May 2012. For the first time, test conductors fired the $\mathrm{J}-2 \mathrm{X}$ in both the secondary and primary modes of operation, 20 seconds in each. Previous tests were run in one mode only; combining the two allowed operators to collect critical data on engine performance.

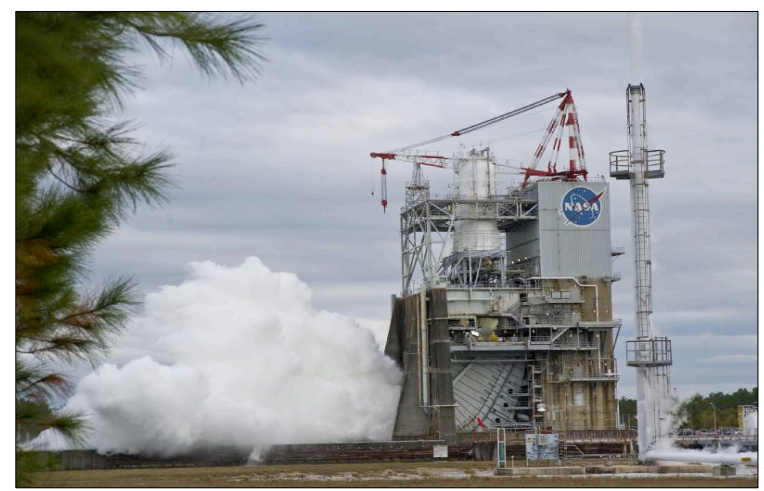

Figure 8 - J-2X 500-sec test firing at SSC

SLS engineers have also modified a J-2X controller to perform closed-loop control of RS-25 hydraulic valves. These tests demonstrate that the controller can simulate a nominal Space Shuttle Main Engine (SSME) flight profile, as well as respond correctly to different failures. By creating a universal rocket engine controller, with minor alterations or configurations to the box, technicians can control multiple engines (including J-2X, RS-25, and F-1) at less than half the cost of an SSME controller. The engines common controller unit (ECU) preliminary design review was successfully completed in October 2012.

In order to produce nearly-finished component parts with almost ideal material properties, a selective laser melting (SLM) machine has been installed at MSFC, with the goal of making rocket engine fabrication more efficient and affordable. It will be used to develop a material properties database for various alloys and, ultimately, hardware for the RS-25 and J$2 \mathrm{X}$ engines. The machine will allow SLS engineers to have greater insight into the SLM process, reducing the cycle time for developing and building parts and reducing per-part costs, thus increasing affordability.

Additionally, NASA is contracting with Boeing to purchase two Delta Cryogenic Second Stages (DCSS) for its first two SLS missions, with options to order two additional upper stages for SLS flights beyond 2021. The DCSS is powered by the same PWR RL-10B2 engine that powers the Delta IV second stage. The DCSS will be modified to be the in-space stage (also known as ICPS) for the first two SLS exploration missions (EM-1 and EM-2) in 2017 and 2021.

The critical path development item for SLS is the massive core stage structure, which will be the largest single stage ever developed for a launch vehicle. The design philosophy of the SLS is to "design once, fly many times," applying the same core systems and structures from the $70 \mathrm{t}$ vehicle to future, larger vehicles including the $130 \mathrm{t}$ super-heavy-lift launcher. Another advantage of the core stage will be that NASA can use existing or proven processes, facilities, personnel, and materials going forward, which will simplify future production as evolved versions of the rocket are rolled out, in keeping with budget and mission requirements.

NASA is also investing in manufacturing hardware and facilities at Michoud Assembly Facility (MAF) in New Orleans, taking advantage of work done by experienced development and operational personnel, as well as a one-of-a-kind infrastructure asset. In particular, SLS is utilizing proven friction stir welding technology at both its MAF and MSFC weld facilities as work on the massive core stage structure ramps up. The SLS Stages Element successfully completed its Core Stage SRR/SDR in June 2012, with Core Stage PDR completed in December 2012.

Wind tunnel testing continues to enhance development of the SLS rocket. Engineers at MSFC's Tri-Sonic Wind Tunnel have put early SLS scale models through more than 900 tests of various crew and cargo configurations. As the design matures, the SLS Program will seek greater understanding of the rocket's aerodynamics by testing on a larger geometric scale at the Langley Research Center (LaRC) Unitary Plan Wind Tunnel in Hampton, VA, as well as at Boeing's Polysonic Wind Tunnel in St. Louis (Figure 9). 


\section{Upcoming Milestones}

In the coming calendar year, SLS will undergo a series of important reviews to ensure its progress toward final design. The PDR will be conducted for the entire vehicle, as will the PDRs for the booster and core stage elements. The SLS Critical Design Review (CDR) is scheduled for early 2014 (Figure 10).

Orion's EFT-1 flight test-with the initial MPCV Stage Adapter providing the launch vehicle

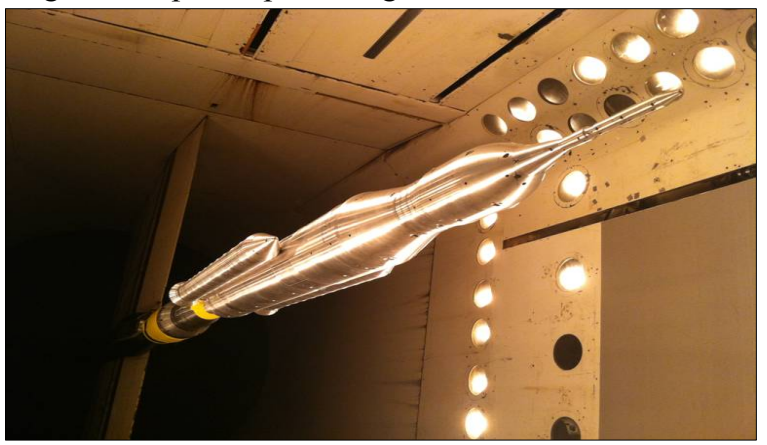

Figure 9 - Wind Tunnel testing continues at MSFC and LaRC interface-will be conducted in early 2014. Also in the 2013-14 timeframe, the GSDO will begin construction of new Vehicle Assembly Building (VAB) platforms for assembling and servicing the SLS.

\section{Platform For EXPANDEd Missions}

The Apollo Program proved the utility of a heavy-lift launch vehicle (HLLV) for human space exploration missions, as it delivers massive capabilities with a single launch, including spacecraft, surface landers/rovers, and propellants for sending these systems to BEO. Both the U.S. and its international partners look forward to the next phase of BEO exploration and have identified an HLLV as important to that effort.

In addition to human exploration activities, SLS also could revolutionize robotic space missions. The primary consideration for most robotic space missions has been the need to fit the payload inside existing launch vehicles, which constrain spacecraft mass and size and often result in complex, origami-

\section{The Road to First Flight in 2017}

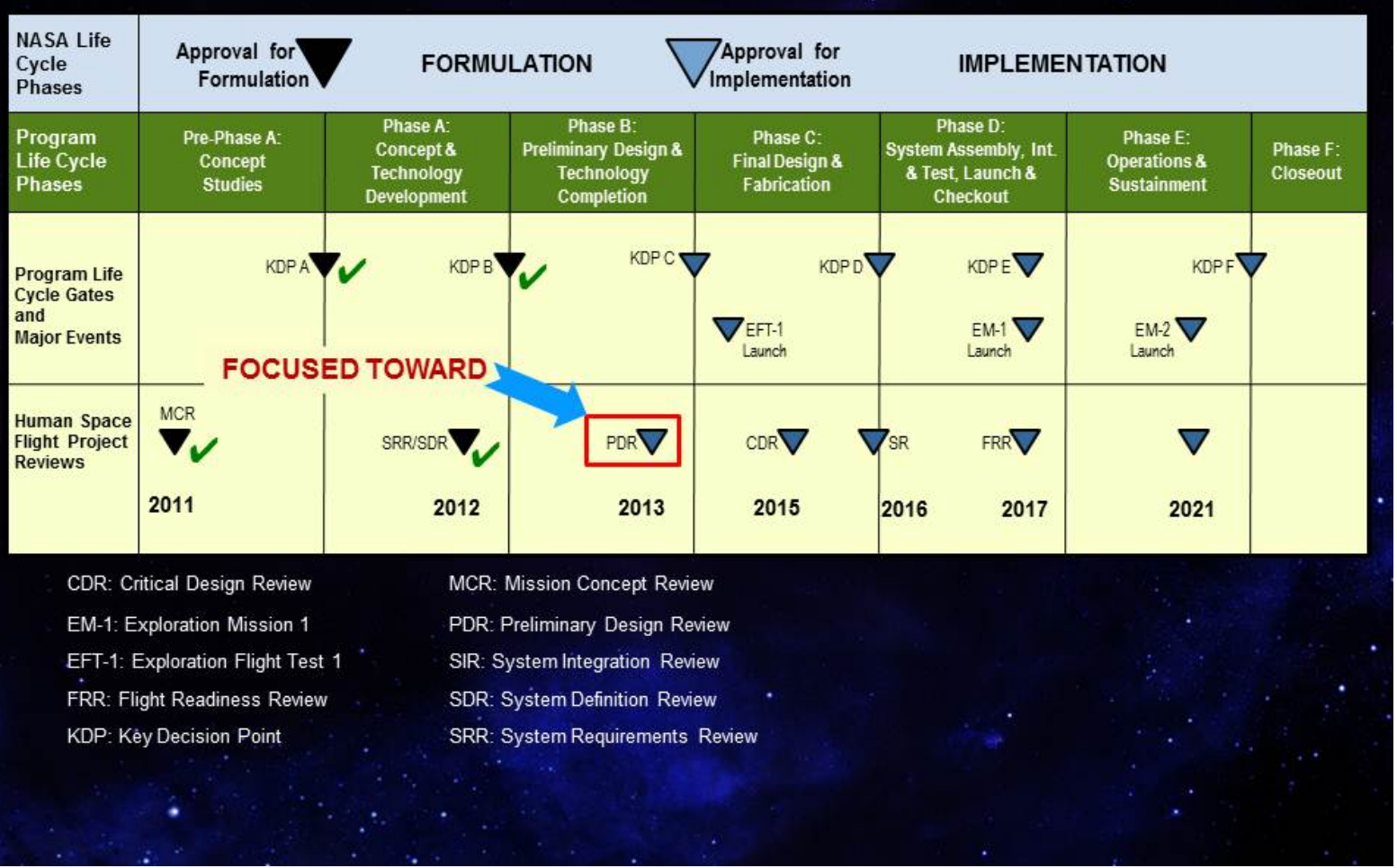

Figure 10 - SLS programmatic milestones 
type folded designs that increase vehicle complexity and risk. SLS provides enough space to allow designers to relax volume constraints and concentrate on developing the instruments necessary to accomplish the primary science mission. Additionally, the significant mass margin provided by SLS allows for a larger propulsion system, reducing complexity of some maneuvers by eliminating gravity assist requirements.

Another constraint for current science missions is the limit on characteristic energy (C3) available to send spacecraft to BEO. The additional energy of SLS offers reduced mission time, thereby reducing power requirements as well as the amount of time that scientific instruments are exposed to space (Figure 11).

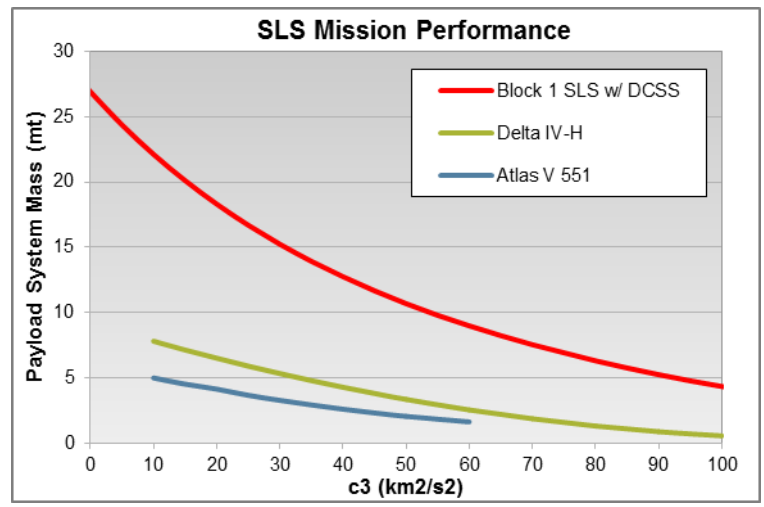

Figure 11 - The C3 curve for the $70 \mathrm{t}$ SLS vehicle shows a decided advantage for science payloads

While commercial launchers have and will continue to serve as the workhorse for many of NASA's science missions, the spacecraft often have to make multiple gravity-assist maneuvers around several of the inner planets before reaching the velocity needed to reach outer planets such as Jupiter or Saturn. These maneuvers increase mission times by years and increase risk to onboard instruments because of the extended time in the space environment.

Primary advantages of SLS to robotic science missions include:

- Volume and mass capability and fewer origami-type payload designs needed to fit in the fairing, leading to increased design simplicity.

- Fewer deployments and critical operations, leading to increased mission reliability and confidence.
- High-energy orbit and shorter trip times, leading to less expensive mission operations.

- Increased lift capacity and payload margin, resulting in less risk.

The SLS team has been discussing the advantages of HLLV with members of the science community. To take advantage of the mass and volume capacity SLS offers, spacecraft designers and mission planners will need to change their fundamental assumptions about what is possible with a single launch vehicle. However, if put to its greatest advantage, SLS could facilitate single-launch missions to the outer solar system, including first-ever sample return missions to Mars, Jupiter/Europa, and Saturn/Titan.

A 2008 National Research Council (NRC) report noted that "NASA should conduct a comprehensive systems-engineering-based analysis to assess the possibility that the relaxation of weight and volume constraints enabled by Ares $\mathrm{V}$ for some space science missions might make feasible a significantly different approach to science mission design, development, assembly, integration, and testing, resulting in a relative decrease in the cost of space science missions." As a heavy-lift vehicle, the SLS offers these advantages. [7]

Building on that philosophy, a recent SLS Utilization Study, conducted as a follow-on to earlier Constellation-era decadal surveys, astronomy workshops, and planetary workshops, investigated arenas of opportunity that extend beyond human exploration goals into other areas of space exploration. [8] The initial process of the study was to perform a literature survey of all potential arenas in order to identify key mission goals and objectives. The literature survey included the various decadal surveys, previous utilization efforts conducted under Project Constellation, and other special studies. Missions were organized and classified into arenas based on their destinations and goals. Over 40 potential mission candidates were identified. [9]

An evaluation of previous mission studies provided the background on exploration goals and objectives. A more detailed examination against key performance benefits (mission trip time, mission complexity, technology risk reduction, payload volume, and mass margin) then identified arenas of opportunity that merited further pursuit.

Identification of these arenas of opportunity was then used to engage potential users of SLS. These potential mission candidates involve multiple customer applications - NASA (human and science 
exploration), commercial, DoD, and international partnerships - as well as multiple arenas, including LEO/highly elliptical orbit (HEO)/geostationary orbit (GEO), cis-lunar/lunar, inner and outer planetary bodies, and asteroids/comets.

Missions that met or exceeded multiple criteria were brought forward as enabling or enhancing missions. Identification of these opportunities provided SLS the information needed to engage potential customers. A summary of these potential missions is captured in Figures 12 and 13. Using agreed-to key parameters, SLS primarily enables or enhances the following missions: Mars Sample Return (MSR), Jupiter Europa Orbiter (JEO), Saturn/Titan Sample Return, Ice Giant Exploration, Outer Planet Sample Return, Large Telescopes, and In-Space Infrastructure. Two of these potential missions will be discussed in more detail in the following sections.

In summary, SLS can provide a unique national capability to enable future exploration goals by reducing mission time, increasing mass margins, reducing spacecraft complexity, and increasing payload volume. With initial screening complete, additional assessments are now underway to further quantify utilization benefits.

\section{Mars Sample Return (MSR)}

Returning a sample from the surface of Mars has been an ambitious goal for the Mars Program for some time. A 2011 National Research Council (NRC) planetary science Decadal Survey concluded that an MSR mission is not only a top science priority, but also a good opportunity to blend the science and human spaceflight elements of NASA. [10]

The SLS Utilization Study identified MSR as a highly regarded potential mission SLS could enable or enhance. Two primary areas that the study focused on were mission complexity and sample size.

A recent Mars Program Planning Group (MPPG) report recognized that the SLS launch cadence and availability may provide a "single shot" MSR opportunity. The MPPG, chartered to provide options that integrate science, human exploration, and technology at an Agency level with Mars exploration as a common objective, found that a sample return orbiter can be integrated into a single launch (of which SLS is a viable option) with a Mars Ascent Vehicle (MAV) lander or combined/co-manifested with other missions. [11]
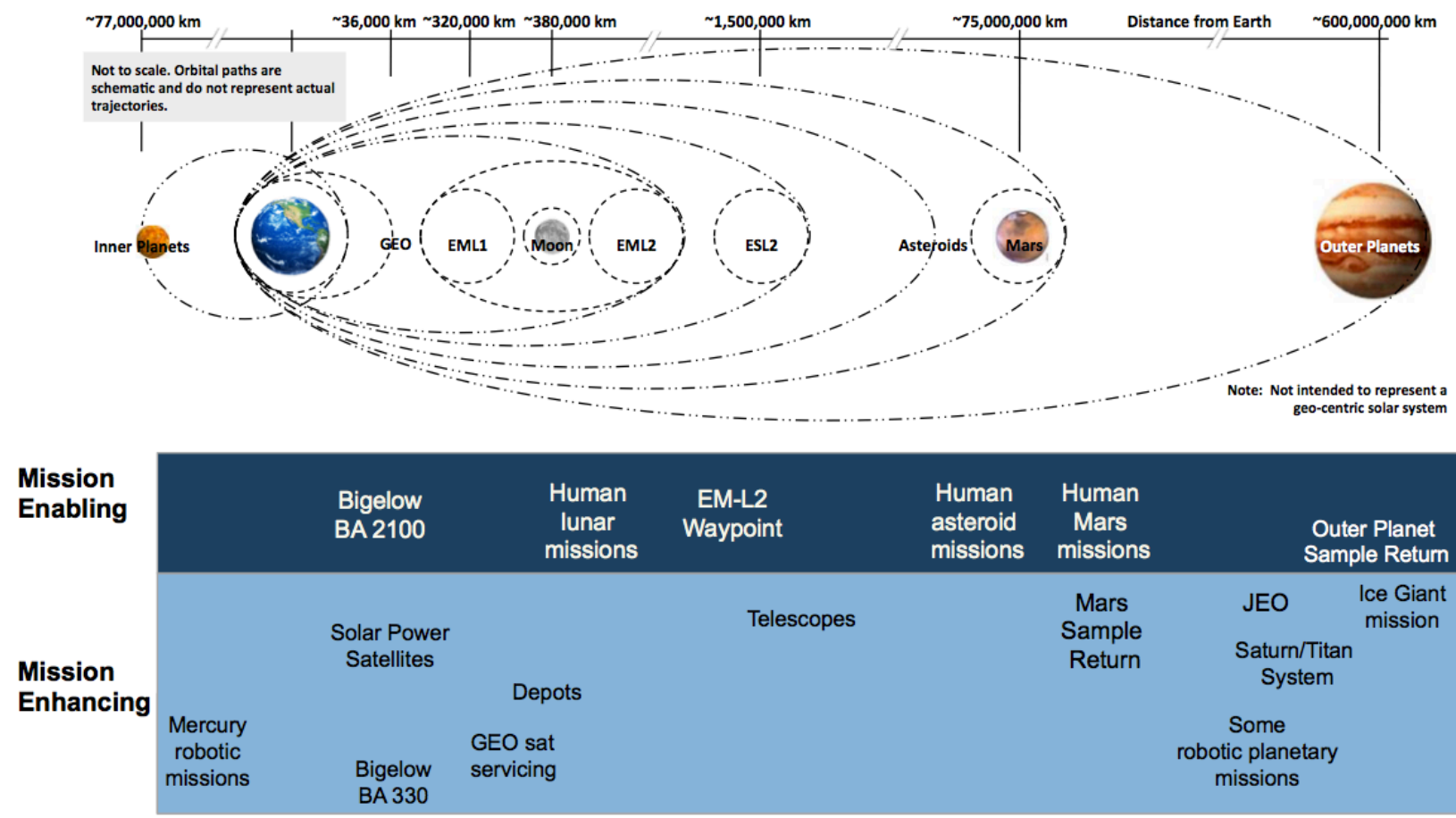

Figure 12 - Summary of SLS mission capabilities 


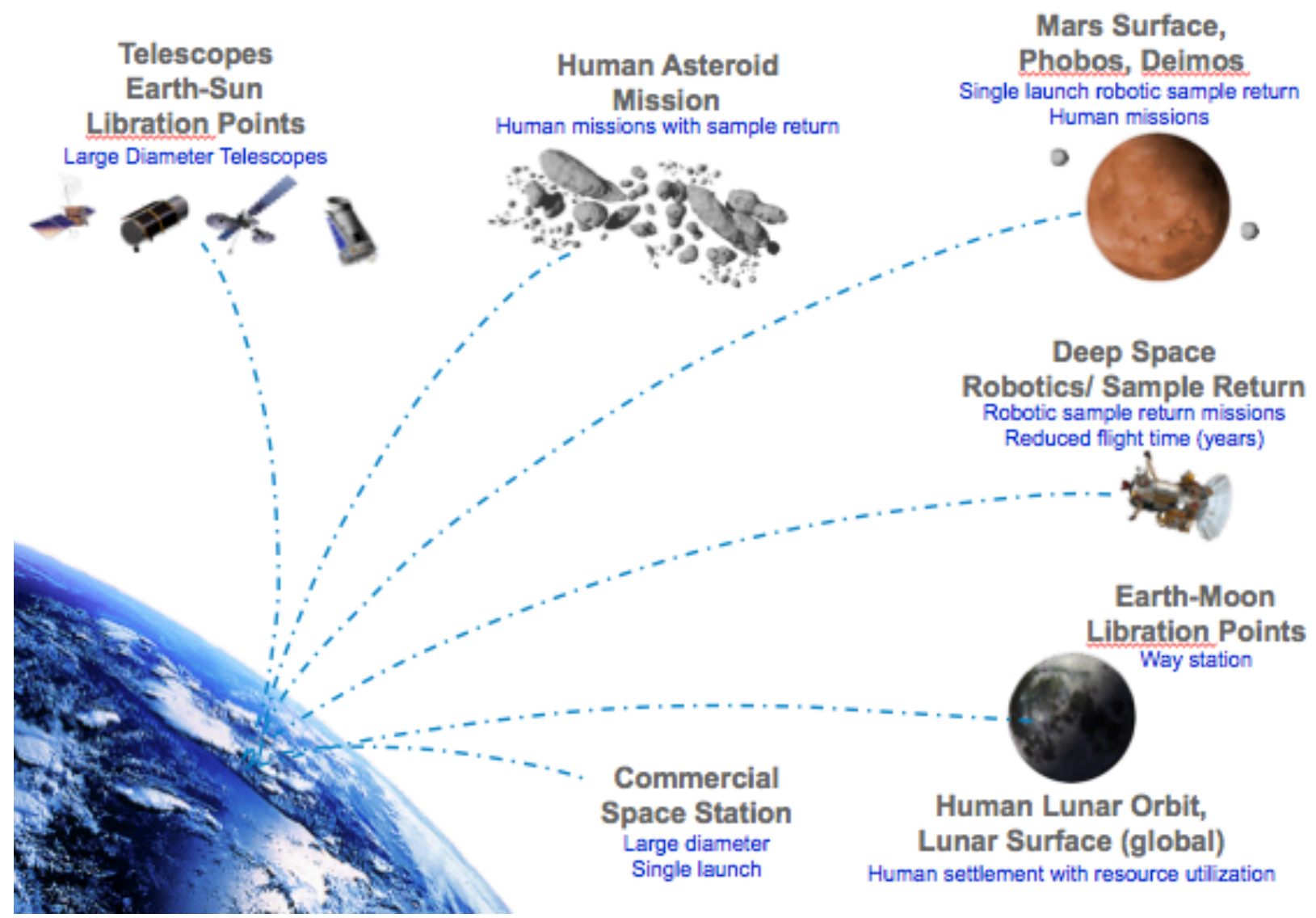

Figure 13 - Missions enabled or enhanced by SLS

Jupiter Europa Orbiter (JEO)

While the primary focus of JEO is to orbit Europa, the science return encompasses the entire Jovian system. The standard trajectory time for a JEO mission ( 6 years) can be cut in half using SLS. Thus, because of the significant mass margin provided by SLS, the Jupiter orbit insertion can be less complex and more flexible. Additionally, a baseline $\sim 5 \mathrm{t}$ spacecraft can be injected to a significantly higher C3, potentially reducing interplanetary trajectory complexity and creating a time-of-flight cost savings. The overall cost savings of using SLS for the JEO mission, factoring in flight time, launch vehicle, radiation mitigation, and spacecraft design, is estimated to be significant.

The JEO mission is extremely complex. Payload mass and size constrain the mission to a complex set of trajectory maneuvers that include Venus-EarthEarth gravity assists, which in turn equate to an almost 9-year mission lifetime. Programmatically, the SLS/JEO partnership leverages significant NASA investment to the benefit of both SLS and JEO, offering significant cost savings to the JEO Program.
Technically, SLS enables quicker return on science due to shorter trip times, increased launch opportunities with high mass margins, more robust radiation shielding due to mass margins, and modular spacecraft design enabling serial hardware development and a smoother funding profile.

\section{SUMMARY}

As NASA continues its role of advancing the frontier, SLS (Figure 14) is providing a unique national capability to enable future exploration goals, offering reduced mission time, increased mass margins, reduced spacecraft complexity, and increased payload volume. It will be a platform for continuing America's tradition of human space flight, as well as providing the capability to launch entirely new science missions and other missions of national and international importance. The benefits of exploration are many: scientific knowledge, technological advancements, economic expansion, public excitement, and national security. SLS is turning plans into progress to deliver on the Nation's commitment to lead both on Earth and in space. 


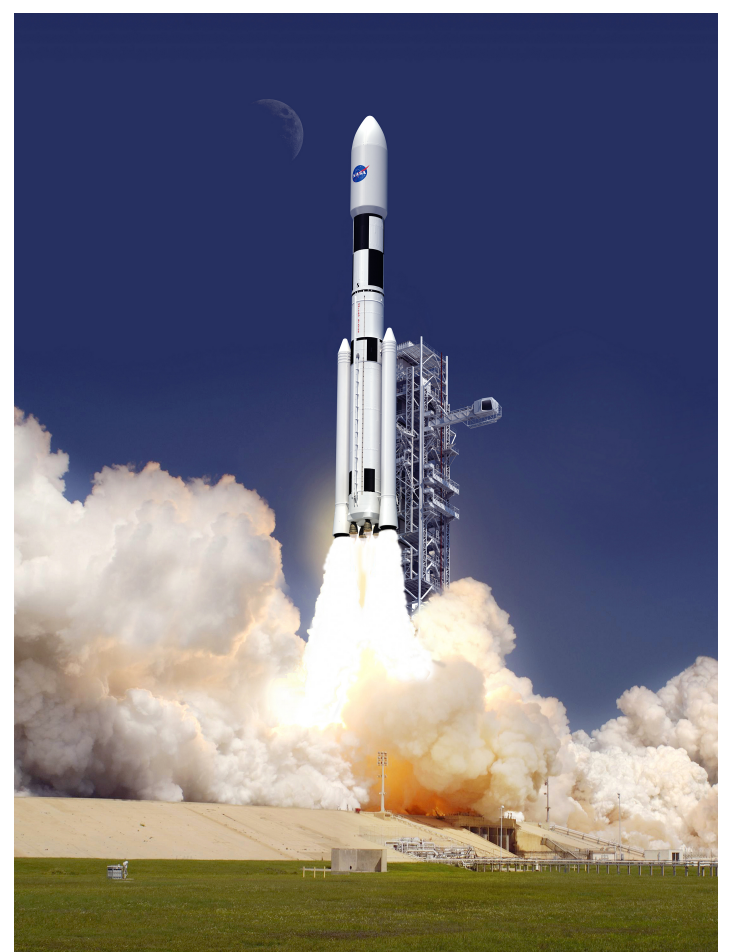

Figure 14 - Artist's concept of SLS 130 t vehicle launching from the Kennedy Space Center

\section{REFERENCES}

[1] NASA Authorization Act of 2010:

http://commerce.senate.gov/public/?a=Files.Serve \&F ile id=20a7a8bd-50f4-4474-bf1d-f0a6a8824b01.

[2] Review of U.S. Human Spaceflight Plans Committee Report, October 22, 2009:

http://www.nasa.gov/pdf/396093main HSF Cmte Fi nalReport.pdf

[3] ATK press release, October 2, 2012:

http://atk.mediaroom.com/2012-10-02-ATK-and-

NASA-Showcase-Cost-Saving-Upgrades-for-Space-

Launch-System-Solid-Rocket-Boosters

[4] NASA press release, October 1, 2012:

http://www.nasa.gov/home/hqnews/2012/oct/HQ 12339_SLS_Awards_Contract.html

[5] NASA press release, September 21, 2012: http://www.nasa.gov/home/hqnews/2012/sep/HQ 12 -333_SLS_Advanced_Development_Proposals.html

[6] NASA Scientific and Technical Information program, "NASA Systems Engineering Handbook,"
2007:

http://ntrs.nasa.gov/archive/nasa/casi.ntrs.nasa.gov/20 080008301 2008008500.pdf

[7] The National Academies Press, "Launching Science: Science Opportunities Provided by NASA's Constellation System," 2008:

http://www.nap.edu/openbook.php?record id=12554 \&page $=\mathrm{R} 1$

[8] NASA Human Exploration and Operations Mission Directorate: "Exploration Planning, Partnerships, and Prioritization Summary," March 6, 2012:

http://www.nasa.gov/pdf/630147main 4Olson Planning\%20Partnerships\%20Priorities\%2003 0612 508.pdf

[9] Morris, Jones; IAC-12.D2.8.4: "Lessons Learned and Work to Date in Launch Vehicle and Payload Design via Early Communications."

[10] National Research Council, "Visions and Voyages for Planetary Science in the Decade 20132022," 2011:

http://solarsystem.nasa.gov/multimedia/downloads/V ision and Voyages-FINAL1.pdf

[11] Mars Program Planning Group Report Summary, September 25, 2012:

http://www.nasa.gov/pdf/691580main_MPPGIntegrated-v13i-Summary\%20Report-9-25-12.pdf

\section{BIOGRAPHY}

Stephen D. Creech is the Strategic Development Manager for the SLS Program, located at NASA's Marshall Space Flight Center in Huntsville, Alabama, where he leads future mission definition, collaboration, and partnerships. He previously served as the Ares $V$ integration manager and

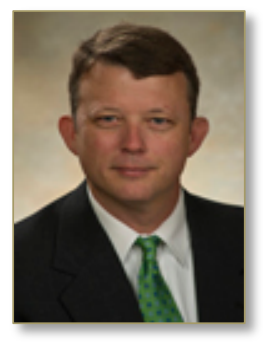
managed the Engineering Cost Group in the Office of Strategic Analysis and Communications at the Marshall Center. Mr. Creech holds a bachelor's degree in Industrial Engineering from Mississippi State University. Honors include NASA's Medal for Exceptional Service and Distinguished Performance Award. 